\title{
Experimental study of turbulence intensity influence for turbulent cross flow in staggered tube bundle using grooved cylinder
}

\author{
Ouissem Sahli ${ }^{1}$, Lahouari Adjlout ${ }^{1, a}$, Omar Ladjedel ${ }^{1}$ and Mohamed amine Ghazi ${ }^{1}$ \\ ${ }^{1}$ Laboratoire d'aérohydrodynamique naval Département de Génie Maritime Mechanical Engineering Faculty USTO MB Oran, Algeria
}

\begin{abstract}
The present work is an experimental investigation on the effect of the turbulence intensity variation in a staggered tube bundle equipped with grooves at $90^{\circ}$ and $270^{\circ}$. The experiments were carried out in a subsonic wind tunnel. Three Reynolds numbers and three turbulence levels were tested. The pressure distributions and drag forces were measured. Surface visualizations were also performed. The obtained results show that the turbulence intensity for different Reynolds number has an influence on the reduction of the drag coefficient.
\end{abstract}

\section{Introduction}

Cross-flow in tube bundles is an active area of research because of its wide practical application in the design for heat exchanger of tubes, offshore structure, cooling system for nuclear plants etc... There has been a lot of experimental and numerical works dedicated to the study of different aspects of flow around cylinders in tandem and to flow in arrays of cylinders. Over the past years, numerous experimental investigations have been carried out to study the complex mechanism of the flow characteristics in different configurations of tube bundles. Aiba et al. [1] performed an experimental study on square in-line tube banks for gap ratio of 1.2 and 1.6. They found that the flow field of the cylinders situated in the second row is relatively affected by the upstream ones because of the absence of vortex shedding behind the first row. Romberg and Popp [2,3] conducted experimental investigations on the stability factors of normal and rotated triangular tube arrays with different pitch-todiameter ratios. Chen and Srikantiah [4] presented series of experiments to measure motion-dependent fluid forces for various tube arrays. Rottmann and Popp [5] further investigated the influence of approach flow on the first row of tube bundles. Žukauskas and Ulinskas [6] reported extensive experimental data for heat transfer and fluid friction during viscous cross-flow in-line and staggered banks of tubes under two thermal boundary conditions. Paul et al. [7] preformed measurements in a staggered tube bundle with fixed pitch-to-diameter ratios at subcritical Reynolds number using the particle image velocimetry technique. Their results showed that the flow exhibits strong Reynolds number dependence in the developing region, however no significant Reynolds number effect could be observed in the spatially periodic region. Paul et al. [8] perform an experimental study on turbulent cross-flow in a staggered tube bundle with transverse and longitudinal pitch-to-diameter ratios of 3.8 and 2.1, respectively. The results reveal higher shear rates in the wake regions.

Although some interesting studies which are based on the premise of modified tube shapes have been carried out, most of the investigations have focused on the characteristics of fluidelastic instability of tube bundles with the circular cylinders. It was found that a cylinder with wavy surface can weaken, to the extent that it may even suppress the vibration comparing with a circular cylinder at the same Reynolds numbers Lam et al. $[9,10]$. An optimal spanwise wavelength ratio for drag reduction and fluctuating lift suppression was found at low Reynolds number of 100 over a wide wavelength range by Lam and Lin [11, 12]. The effects of introducing a row of wavy cylinders with suitable wavelength into circular tube bundles for the control of flow-induced vibration were investigated by $\mathrm{K}$. Lam et al. [13], two types of tube bundles with the first row of circular tubes replaced by different wavy cylinders are studied and the results are compared with that of purely circular cylinders at the Reynolds number of 7500. Because of the introduction of this kind of wavy tubes at the first row, the turbulent kinetic energy behind the first row tubes are reduced and the wake patterns of the second row tubes are better organized compared with the tube bundle with purely circular cylinders and the tube bundle with wavy cylinders placed in the first row. The power density spectra of the vortex shedding in the tube bundle are evidently suppressed. This implies that the turbulent forces on the second tube row are weakened. It is concluded that by introducing a suitable wavy cylinders in tube bundles, some good effects on the control of flow-

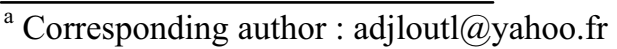


induced vibration of the tube bundles in the heat exchanger can be achieved. Ladjedel et al. [14] performed an experimental investigation using grooved cylinders in in-line tube bundles configuration. They found a reduction of $36.5 \%$ in the pressure drop for a Reynolds number of $1.33 \times 10^{4}$.

Traub [15], has studied the influence of turbulence intensity on the heat transfer and the pressure drop in plain tube bundles. The results of this study showed that an increase of the turbulence intensity from $0.8 \%$ to $25 \%$ leads to an increase of the Nusselt number about $42 \%$ for a single row and $14 \%$ for a tube bundle with three rows. Furthermore, Traub [15] presented the results for the enhancement of heat transfer according to the criterion of Stephan and Mitrovic [16]. The dimensionless pressure drop coefficient is related to the Reynolds number and to the turbulence intensity level of the external flow around the heat exchanger tubes. The Reynolds number of the flow through the heat exchanger is calculated using a characteristic length of the heat exchanger, which is usually either the tube diameter or the minimum distance between the tubes inside the heat exchanger and the maximum velocity of the flow passing through the heat exchanger, Umeda and Yang [17]. In previous studies it has been observed that for high Reynolds number and turbulence levels the pressure drop decreases. Beziel and Stephan [18] concluded to this observation for a range of Reynolds numbers from 4000 to 200,000 and for turbulence intensities from $0.8 \%$ up to $38.8 \%$. Regarding the heat transfer mechanism, Sak et al. [19] studied the role of the turbulence length scale and intensity on forced convection from a heated horizontal circular cylinder. Different levels of turbulence were imposed by a grid located in the inlet section of the flow field which had the role of turbulence generator. They derived an expression correlating the Nusselt number with the Reynolds number and the turbulence intensity. They showed that an increase of Reynolds number and turbulence intensity leads to higher Nusselt numbers

Experimental investigation is performed; the forces on the cylinders are measured using a TE 81 balance and a TE44DPS differential pressure with a multichannel scanner. The purpose of the present work is to investigate experimentally the influence of the turbulence intensity on staggered configuration equipped with grooved cylinders.

\section{Experimental set-up}

The experiments are carried out in closed circuit wind tunnel. The test section is of square cross-section $0.46 \mathrm{~m} \times$ $0.46 \mathrm{~m}$ and $1.2 \mathrm{~m}$ long. The closed circuit wind tunnel is of conventional design in which the velocity could reach 60 $\mathrm{m} / \mathrm{s}$ and the turbulence intensity is less than $1 \%$. The test section is composed of 17 rigid PVC tubes of an identical external diameter of $40 \mathrm{~mm}$ and $460 \mathrm{~mm}$ length arranged in a staggered configuration as shown in Figure 1. Half tubes are also mounted along the bottom and top walls of the test model alternately to simulate an infinite tube bundle and minimize the wall boundary layer. All the tube arrays are investigated in this study. A schematic diagram of the arrangement is shown in Figure 2.

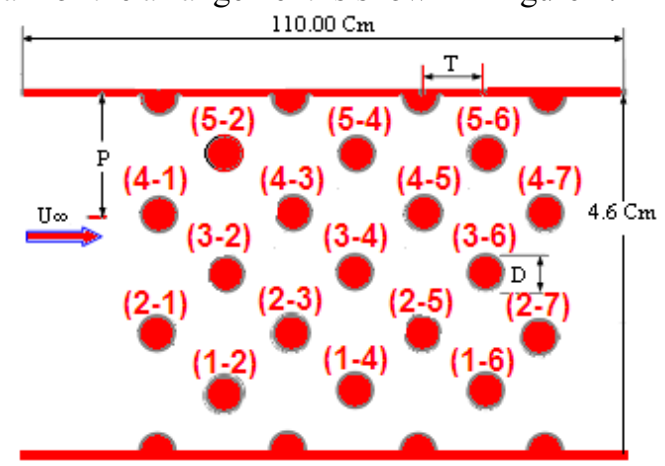

Figure 1. The geometrical features of the tube bundle.

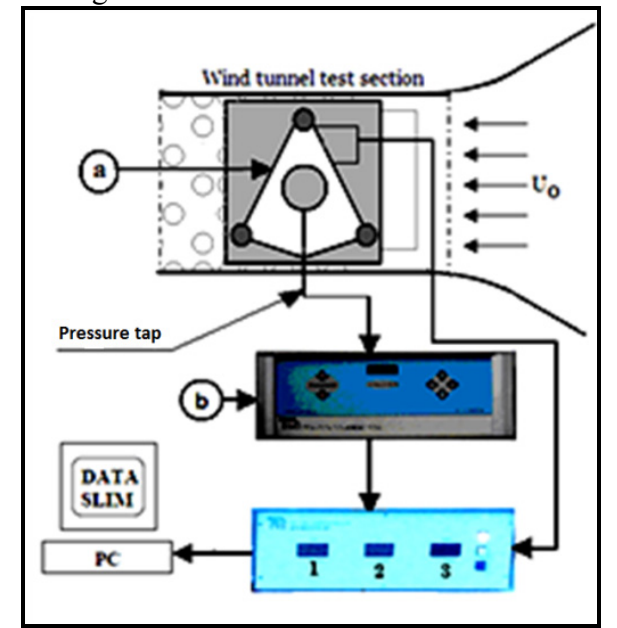

Figure 2. Measurement setup of drag and pressure. a: balance TE81, b: TE44 DPS

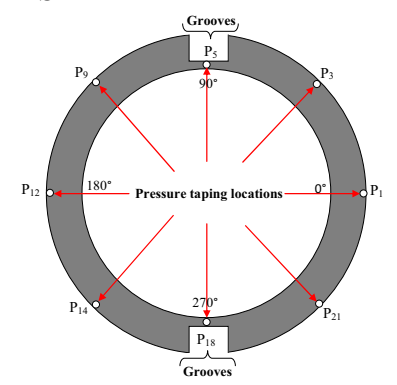

Figure 3. Pressure taping locations.

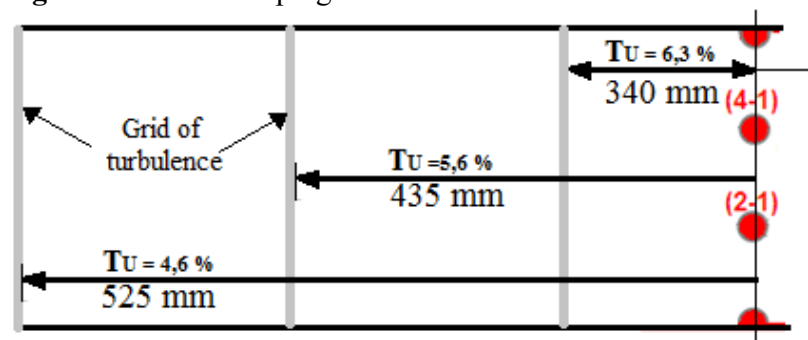

Figure 4. turbulence level generated for each position of the turbulence grid.

The experiment setup is shown in Figure 2. The tubes in the array are rigidly fixed, except for one tube which will be referred to as the instrumented cylinder equipped with 22 pressure tapings on its circumference at midspan. The shape of the groove and the pressure tapings position are shown in Figure 3. The diameter of the pressure tapings is $0.5 \mathrm{~mm}$. The pressure measurements 
are carried out for the 17 tubes for an azimuthal angle from $0^{\circ}$ to $360^{\circ}$. The pressure distributions on the cylinder are measured using a TE44DPS differential pressure with a multi-channel scanner. The latter pressure scanning box allows sequential selection of up to 20 pressure tapings. The display unit links to a computer, loaded with DATASLIM software for data analysis and logging of the results. To generate turbulence Roach [20] method was adopted. The latter uses different turbulence grids. In this study the SMS (square mesh arrays of square bars) type is chosen (figure 4).

\section{Result and discussion}

In order to investigate the change of flow characteristics around staggered tube bundle by the use of the grooved cylinders the experiments are performed for the following physical parameters:

- Three Reynolds number are studied $\mathrm{Re}=9300, \mathrm{Re}=$ 13950 and $\mathrm{Re}=20000$ (based on the free stream velocity).

- Pitch to diameter ratio, $\mathrm{P} / \mathrm{D}=3.8$ and $\mathrm{T} / \mathrm{D}=2.1$.

However, visualizations are carried out for selected Reynolds numbers.

The measurement of pressure distribution starts from the frontal point facing the mainstream flow and ends at the same point after a complete revolution. The uncertainty in the pressure measurement was estimated to be less than $\pm 5 \%$ and for the drag and lift forces less than $\pm 2.4 \%$

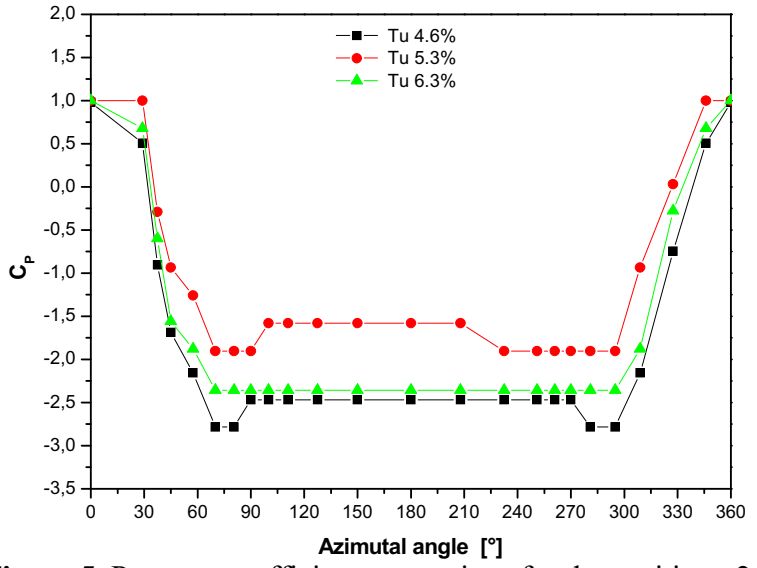

Figure 5. Pressure coefficient comparison for the position 2-1 $(\operatorname{Re}=9300)$.

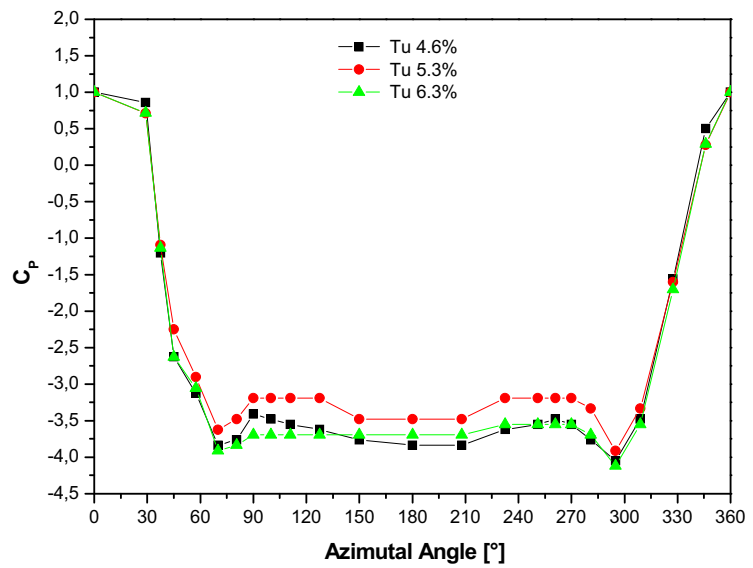

Figure 6. Pressure coefficient comparison for the position 3$2(\mathrm{Re}=13950)$.

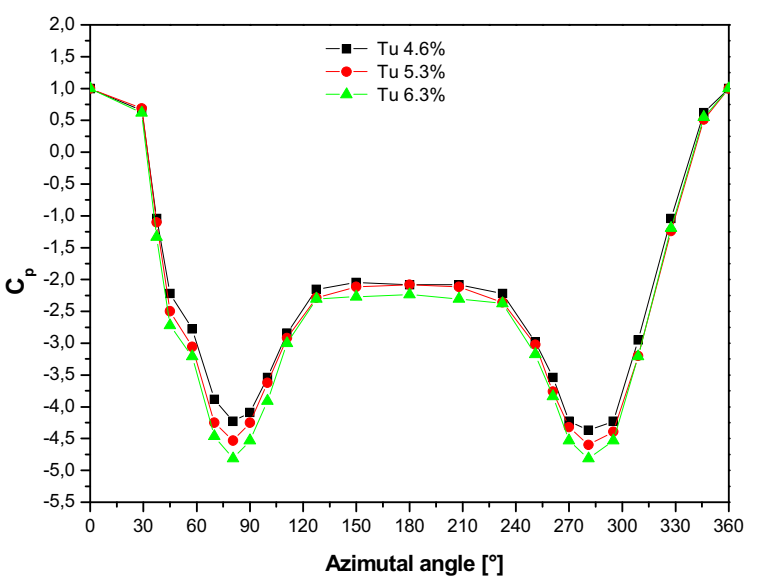

Figure 7. Pressure coefficient comparison for the position 4$3(\mathrm{Re}=20000)$.

Figure 5, 6 and 7 show the pressure coefficient distribution around the cylinders (2-1), (3-2) and (4-3) respectively at different Reynolds numbers and turbulence intensities $(\mathrm{Tu})$. It is observed that the curves have the same trend where the flow around the cylinders starts from the stagnation point $\left(\theta=0^{\circ}\right)$ in which $\mathrm{Cp}$ takes a value of one. The flow speeds up then starts to increase accompanied by a drop in the $\mathrm{Cp}$ to negative values. The flow velocity begins to reduce near $\theta=60^{\circ}$ along with an increment of pressure in the direction of the flow, which results in the adverse pressure gradient situation. The flow now has to move against the pressure force in addition to the viscous force. This leads to a reduced gradient of the velocity profile and the wall shear stress. Separation of flow occurs when the shear stress cannot overcome the adverse pressure gradient, this happens at $\theta=75^{\circ}$ for the tube $2-1, \theta=70^{\circ}$ for the tube $3-2$ and at $\theta=85^{\circ}$ for the tube $4-3$. It is clearly seen in figure 5 and 6 that the pressure drag is more reduced due to the observed reduction in the pressure on the downstream of the cylinder for $\mathrm{Tu}=5.3 \%$ compared to the other levels

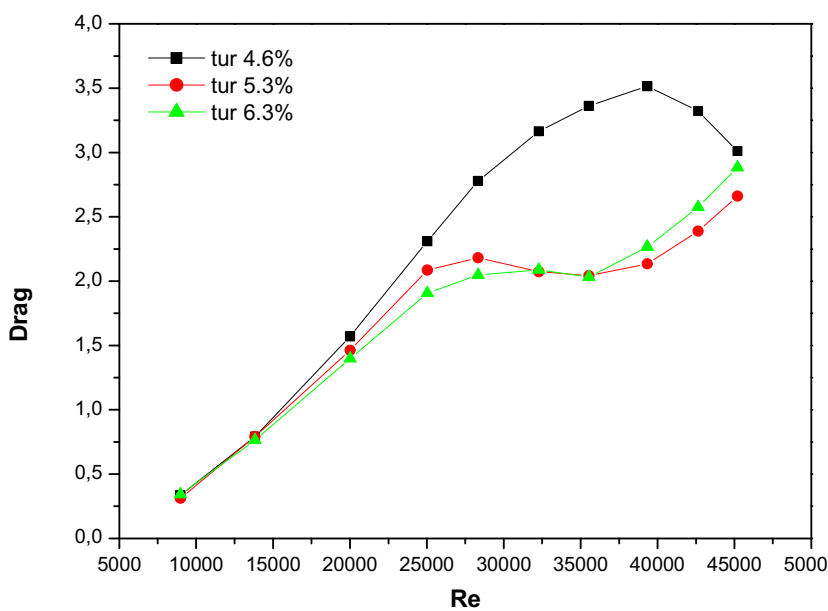

Figure 8. Drag force distribution along the tube length for different Reynolds number and turbulence intensities. (tube 5-2)

Figure 8 shows the drag force evolutions for different Reynolds number and turbulence intensities for the position 5-2. It is noted that the curves of the drag force distribution for the two levels 5,3 and $6,3 \%$ have the 
same trends; but at a critical Reynolds number of 25000 the trend of the level $\mathrm{Tu}=4,6 \%$ is not behaving in the same way. This indicates an influence of the level of turbulence. For level $\mathrm{Tu}=4.6 \%$ arriving at $\mathrm{Re}=40000$, the strength of the drag decreases. This phenomenon is explained by the surface visualization for $\mathrm{Re}=45000$ of figure 9 , where the separation point is situated after the groove. Therefore a reduced wake distance resulting in a lower drag force is obtained.

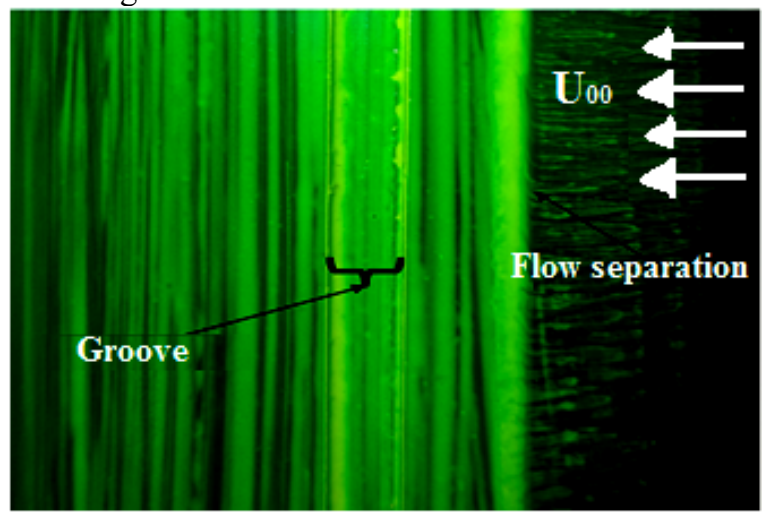

(a) $\operatorname{Re}=25000$

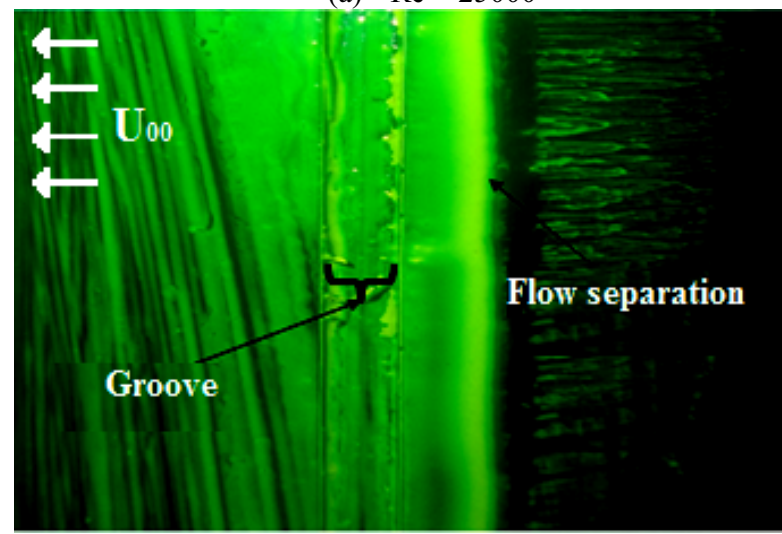

(b) $\mathrm{Re}=35000$

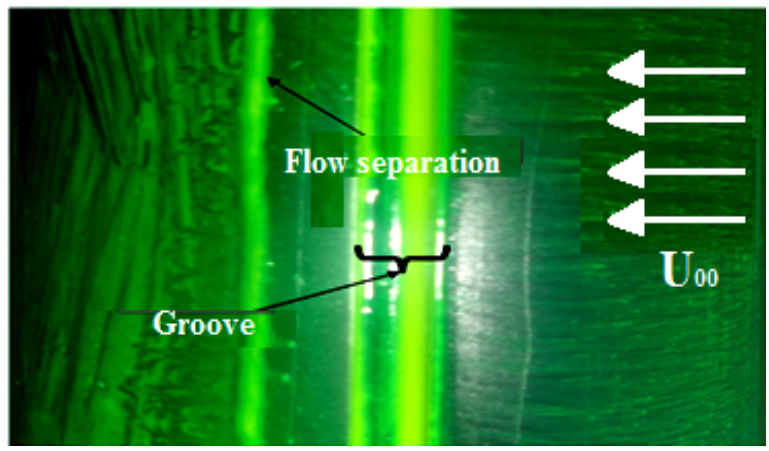

(c) $\mathrm{Re}=45000$

Figure 9. Surface flow visualization for $\mathrm{Tu}=4.6 \%$ (tube 5-2).

From $\mathrm{Re}=25000$, the drag force decreases. Compared to $\mathrm{Tu}=4.6 \%$, the drag force has lower values for $\mathrm{Tu}=6.3 \%$. The visualization shows that when exceeding a number of $\mathrm{Re}=25000$, the flow separation is close to the groove which leads to the decrease in the drag force value.

Consequently, it is noted that by increasing the turbulence intensity the separation angle is delayed causing a decrease in the value of the drag.

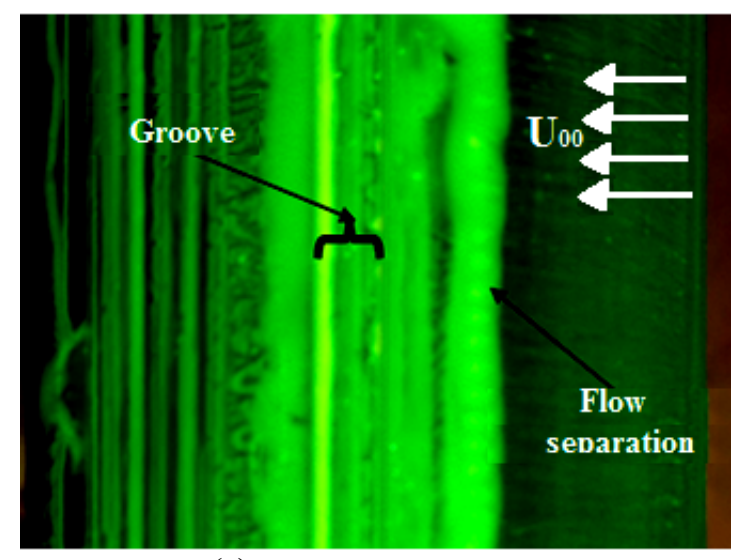

(a) $\quad \operatorname{Re}=25000$

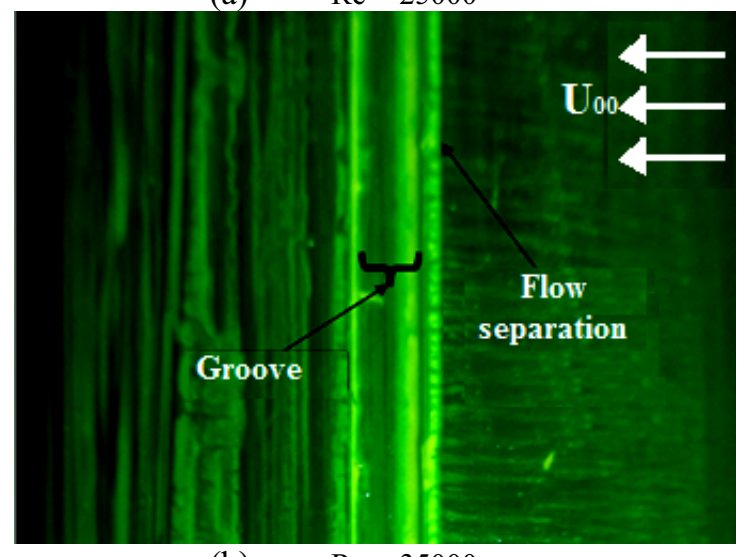

(b) $\operatorname{Re}=35000$

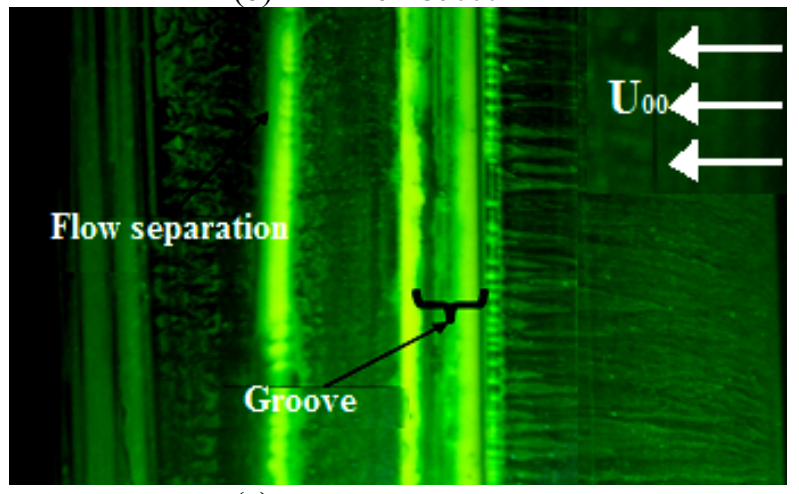

(c)

$\operatorname{Re}=45000$

Figure 10. Surface flow visualization for $\mathrm{Tu}=6.3 \%$ (tube 5-2).

\section{Conclusion}

The turbulent cross flow in staggered tube bundle arrangement with transverse and longitudinal pitch-todiameter ratios of 3.8 and 2.1 respectively is experimentally investigated. The configuration is equipped with grooved cylinders. The obtained results seem to agree rather well with the experimental and theoretical data already gathered, in particular on the distribution of pressure around the examined cylinders. The following conclusions are drawn:

- Increasing the turbulence level delayed the separation point;

- At some critical Reynolds number, increased turbulence level leads to a decrease in the drag force in column 2; 
- By increasing the Reynolds number of the separation point is delayed.

- The evaluation and analysis of the distribution of the pressure on the surface of the cylinder; it is found that the pressure drag reduction is due to the decreasing pressure in the front face of the circular cylinder and the moving aft of the separation point on the circular cylinder.

Flow visualization provided a clear picture of the separation point which can help in better understanding of the flow character.

\section{References}

1. S. Aiba, H. Tsuchida, T. Ota. Bull JSME, 25, 919 (1982).

2. O. Romberg, K. Popp. J. Fluid Struct., 12, 17 (1998a).

3. O. Romberg, K. Popp. J. Fluid Struct, 12, 153 (1998a).

4. S.S. Chen, G.S. Srikantiah. ASME J. Press. Vess. Technol., 123, 429 (2001).

5. M. Rottmann, K. Popp. J. Fluid Struct, 18, 595 (2003).

6. A. Žukauskas,R. Ulinskas. Heat Transfer in Tube Banks in Crossflow, Hémisphère, New York. (1988).

7. S.S. Paul, M.F. Tachie, S.J. Ormiston. Int. J. Heat Fluid Flow, 28, 441 (2007).

8. S.S. Paul, S.J. Ormiston, M.F. Tachie. Int. J. of Heat and fluid flow, 29, 387 (2008).

9. K. Lam, F.H. Wang, J.Y. Li, R.M.C. So. J. Fluid. Struct., 19, 321 (2004a).

10. K. Lam, F.H. Wang, R,M.C. So. J. Fluid Struct., 19, 815 (2004b).

11. K. Lam, Y.F. Lin. J. Mech. Sci. Technol., 21, 1331 (2007)

12. K. Lam, Y.F. Lin. Fluid Mech., 620, 195 (2009).

13. K. Lam, F. Lin, L. Zou, Y. Liu. Int. Journal of Heat and Fluid Flow, 31, 32 (2010).

14. O. Ladjedel, L. Adjlout, T. Yahiaoui, O. Imine. Mechanics \& Industry, 14, 287 (2013).

15. D. Traub, Chem. Eng. Process. 28, 173 (1990).

16. K. Stephan, J. Mitrovic, Chem. Ing.Technol. 56, 427 (1984).

17. S. Umeda, S.W. Yang. Exp. Fluids 26, 389 (1999).

18. M. Beziel, K. Stephan. Chem. Eng. Technol. 15, 219 (1992).

19. C. Sak, R. Liu, D.S.-K. Ting, G.W. Rankin. Exp. Thermal Fluid Sci. 31, 279 (2007).

20. P. E. Roach 'the generation of nearly isotropic turbulence by means of grids". Vol 8, $\mathrm{N}^{\circ} 2$, June (1987). 